\title{
ALTERNATIVAS PARA A EXPERIMENTAÇÃO INDUSTRIAL EM ESQUEMA FATORIAL: UM ESTUDO DE CASO PARA ADESIVO ESTRUTURAL DE BASE ACRÍLICA
}

\section{ALTERNATIVES TO INDUSTRIAL FACTORIAL EXPERIMENTS: A CASE STUDY FOR STRUCTURAL ACRYLIC-BASED ADHESIVE}

\author{
Juliano Bortolini ${ }^{1}$; Walter Accioly Costa Porto ${ }^{2}$; Marcelo Silva de Oliveira ${ }^{3}$ \\ ${ }^{1}$ Universidade Federal de Mato Grosso - UFMT - Cuiabá - Brasil \\ julianobortolini@ufmt.br \\ ${ }^{2}$ Instituto Federal da Bahia - IFBA - Salvador - Brasil \\ walterporto@hotmail.com \\ ${ }^{3}$ Universidade Federal de Lavras - UFLA - Lavras - Brasil \\ marcelo.oliveira@dex.ufla.br
}

\begin{abstract}
Resumo
Experimentos são realizados pelas indústrias com o intuito de aumentar a compreensão de seus processos de fabricação. Numa época em que a indústria brasileira vê a necessidade de equipararse às indústrias competitivas de países desenvolvidos e emergentes, não se pode prescindir da apreciação realista da adoção de técnicas de gestão industrial voltadas à experimentação, as quais visam à melhoria da produtividade e da qualidade de processos e produtos. Desta forma, objetivase neste artigo abordar o planejamento e análise de experimentos fatoriais $2^{k}$ no contexto industrial. Especificamente, propôs-se expor aspectos e conceitos básicos de experimentação e sugerir, como alternativa para o uso da técnica de experimentação um-fator-por-vez, o planejamento de experimentos fatoriais $2^{k}$ completos, que são úteis quando se tem por finalidade examinar um grande número de fatores para determinar quais são os mais importantes, ou quando o tempo ou o recurso para o experimento são escassos. As técnicas do confundimento $e$ experimento não repetido também são abordadas. A primeira é uma alternativa para experimentos em que há dificuldade em manter constante alguma característica do processo produtivo, e a segunda quando é inviável realizar repetições. Como resultado ilustram-se as técnicas estatísticas apresentadas, sendo essas aplicações adaptadas de um mesmo experimento de verificação da influência de quatro fatores na resistência mecânica de junções adesivas.
\end{abstract}

Palavras-chave: confundimento; experimento industrial; experimento não repetido; um-fator-porvez.

\section{Introdução}

O interesse e a necessidade das indústrias de desenvolver e melhorar processos de fabricação - tendo como um dos objetivos a melhoria da produtividade e da qualidade de seus produtos - tem suscitado a utilização de técnicas estatísticas, em especial o planejamento e análise de 
experimentos, pelas indústrias. Numa época em que a indústria brasileira vê a necessidade de equiparar-se às indústrias competitivas de países desenvolvidos e emergentes, não se pode prescindir da apreciação realista da adoção de técnicas de gestão industrial voltadas à experimentação, as quais visam à melhoria da produtividade e da qualidade de processos e produtos.

Por outro lado, de forma preocupante, Tanco et al (2008) salientam que na literatura há uma reunião de evidências e dúvidas, apresentadas em estudos de casos, sobre a integridade dos planejamentos e análises de experimentos conduzidos pelas indústrias.

Uma das explicações possíveis para esse cenário, planejamento inadequado de experimentos, segundo Czitrom (1999), é a falta de considerações práticas do planejamento de experimentos nos cursos acadêmicos de Engenharia.

Outra explicação cabível é a escassa relação entre universidade e empresa. Segundo Rapini (2007), há pouco interesse das empresas em estabelecer relações com as universidades. E quando as cooperações estão presentes, estas limitam-se a atividades de consultoria.

Para um planejamento adequado de um experimento industrial, espera-se que as peculiaridades típicas da indústria sejam consideradas. Destacam-se algumas dessas peculiaridades:

a) dificuldade de interromper o processo produtivo. Nesse caso são sugeridos os experimentos denominados online. Em relação a esses experimentos, Box (1957) propôs o Evolutionary Operation (EVOP), um método de controle e melhoria de processos a ser usado durante o processo produtivo;

b) complicação, ou até mesmo a impossibilidade, de modificar constantemente algumas características do processo industrial. Nesta situação, sugere-se o uso de parcelas subdivididas. Jones e Nachtsheim (2009) e Goos, Langhans e Vandebroek (2006), abordam esses experimentos, e ressaltam que muitos experimentos em parcelas subdivididas são analisados como se não o fossem;

c) obstáculo em manter constante alguma característica do processo produtivo - por exemplo, matérias primas de um mesmo fornecedor. Nessa situação é recomendado o uso do delineamento em blocos casualizados, abordado neste artigo;

d) inviabilidade em realizar repetições por causa, por exemplo, do alto custo envolvido no processo. Para essa inviabilidade aconselha-se o experimento fatorial não repetido, abordado, também, neste artigo.

Ilzarbe et al (2008), em revisão de casos publicados em revistas científicas entre os anos 2001 e 2005, constataram que a maioria dos experimentos é proveniente das indústrias de materiais, mecânica e química. Sendo que a maioria deles investiga se certas condições, ou configurações, de 
um processo influenciam em algumas características dos produtos resultantes desse processo, ou seja, são experimentos de triagem.

Neste artigo serão abordados alguns aspectos do planejamento e análise de experimentos e algumas técnicas da estatística experimental que podem ser úteis para algumas particularidades dos experimentos em processos industriais, em especial quando o problema de custo e esforço é limitante.

As técnicas experimentais abordadas, tais como fatorial $2^{k}$ completo não repetido e confundimento, são apropriadas para a fase de triagem de variáveis influentes. Essas técnicas serão ilustradas através de um experimento de certificação da influência de quatro fatores na resistência mecânica de junções adesivas. Por fim, são feitas breves considerações acerca dos experimentos fatoriais $2^{k}$.

\section{Planejamento e análise de experimentos industriais}

Experimento, de acordo com Montgomery (2009), é um teste, ou série de testes, em que são feitas mudanças propositais em algumas características do processo, de modo a observar e identificar as razões das mudanças que puderam ser observadas em uma variável resposta, que, segundo Mason, Gunst e Hess (2003), é simplesmente uma observação do experimento.

Um processo pode ser esquematizado conforme o modelo da Figura 1, em que as variáveis de entrada controláveis $x_{1}, x_{2}, \cdots, x_{p}$, segundo Montgomery (2009), podem ser visualizadas como uma combinação de máquinas, métodos, pessoas e outros recursos que transformam entrada (geralmente materiais) em saída, que tem uma ou mais variáveis respostas. As variáveis de entrada não controláveis $z_{1}, z_{2}, \cdots, z_{q}$ são características experimentais não controladas, seja por desconhecimento da existência delas ou pelo alto custo para controlá-las. Tais variáveis constituem o erro experimental.

Figura 1 - Representação de um modelo geral de processo

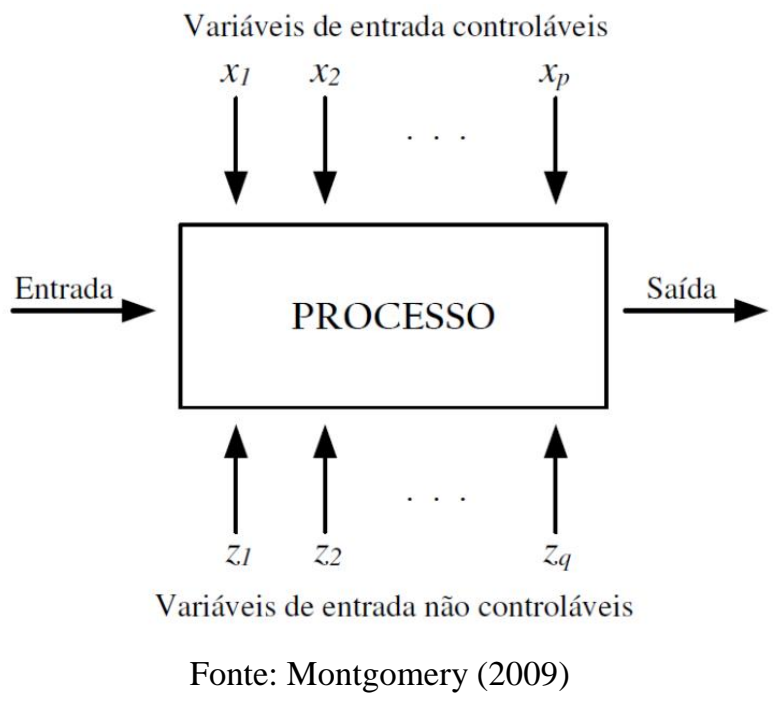


É importante notar que uma variável de entrada é considerada controlável se os valores que ela pode assumir, denominados níveis, podem ser determinados antes do início dos testes. As variáveis de entrada controláveis que são de interesse em serem investigadas pelo pesquisador, geralmente, são denominadas de fatores, e as combinações possíveis entre os níveis dos fatores são chamadas de tratamentos.

Antony (2003) menciona que, em processos de fabricação, é comum o interesse em conduzir experimentos para explorar relações entre variáveis de entrada e de saída de um processo. No entanto, alerta que, para um experimento ser válido, é necessário valer-se de abordagem científica, a fim de planejá-lo e analisá-lo. Na estatística experimental, o planejamento de experimentos estatísticos refere-se ao processo de realizar um experimento, de tal forma que, os dados obtidos possam ser analisados por meio de métodos estatísticos, e levem a conclusões válidas (MONTGOMERY, 2009).

Entre os métodos estatísticos considerados na estatística experimental destaca-se a análise de variância (ANAVA), desenvolvida por Ronald Aylmer Fisher (1890 - 1962), que permite verificar se há diferenças significativas entre as variáveis consideradas, denominadas também de fontes de variações. Outras técnicas estatísticas também são utilizadas pelas indústrias, como as da estatística multivariada e a metodologia de superfície de resposta. Bakke, Leite e Silva (2008) revisam as técnicas multivariadas, em especial a análise fatorial, na engenharia de produção. Lima, Balestrassi e Paiva (2011) e Galdamez e Capinetti (2004) utilizam a metodologia de superfície de resposta na otimização de um processo industrial.

Segundo Cochran (1947), a ANAVA depende dos seguintes pressupostos:

a) que os efeitos das variáveis de entrada, controláveis e não controláveis, sejam aditivos;

b) que os erros experimentais sejam independentes, normalmente distribuídos de média nula e variância constante.

O desenvolvimento teórico da ANAVA, tal como a demonstração de alguns teoremas, são apresentados em Irwin (1931).

Para diligenciar que os pressupostos da ANAVA sejam satisfeitos e assegurar que as análises estatísticas sejam válidas, há três princípios básicos na experimentação. São eles: repetição, aleatorização e controle local (FISHER, 1971).

De acordo com Montgomery (2009) e Mason, Gunst e Hess (2003), repetição é duas ou mais observações obtidas para determinada combinação específica de níveis dos fatores, os quais são conduzidos, tanto quanto possível, sob idênticas condições experimentais. 
Conforme Montgomery (2009) e Hinkelmann e Kempthorne (2008), o princípio da repetição tem por finalidade propiciar a obtenção de estimativa do erro experimental.

O princípio da aleatorização, segundo Hinkelmann e Kempthorne (2008), consiste em atribuir a todos os tratamentos a mesma probabilidade de serem designados a qualquer unidade experimental, que é a entidade na qual uma observação é feita, e tem por finalidade proporcionar uma estimativa válida para o erro experimental.

O ideal em um experimento é que todas as unidades experimentais sejam homogêneas, isto é, que não haja diferença sistemática entre elas. Devem ser tão iguais quanto possível em todas as características que possam afetar a resposta (WU; HAMADA, 2009; MASON; GUNST; HESS, 2003). No entanto, nem sempre será possível obter unidades experimentais homogêneas. Em tais situações, faz-se uso do terceiro princípio da experimentação, o controle local.

A ideia básica do controle local consiste em dividir o número total de unidades experimentais em dois ou mais grupos, de maneira que as unidades de cada grupo sejam homogêneas entre si (WU; HAMADA, 2009; HINKELMANN; KEMPTHORNE, 2008; MASON; GUNST; HESS, 2003). O termo bloco geralmente é usado para se referir a um conjunto de unidades experimentais homogêneas.

O modo como é feita a aleatorização dos tratamentos nas unidades experimentais estabelece os delineamentos experimentais, sendo os delineamentos inteiramente casualizado e em blocos casualizados os mais comuns na experimentação industrial. Na Tabela 1 há um esquema com os delineamentos supracitados e as suas características, situações apropriadas na engenharia de produção e bibliografias.

Tabela 1 - Algumas características, situações apropriadas e bibliografias para o delineamento inteiramente casualizado e em blocos casualizados

\begin{tabular}{ccccc}
\hline Delineamento & Característica & Situação apropriada & Bibliografia \\
\hline $\begin{array}{c}\text { Delineamento } \\
\text { inteiramente } \\
\text { casualizado } \\
\text { (DIC) }\end{array}$ & $\begin{array}{c}\text { Não há restrição na } \\
\text { aleatorização dos } \\
\text { tratamentos }\end{array}$ & $\begin{array}{c}\text { Quando todas as unidades experimentais podem ser } \\
\text { consideradas homogêneas. Tais como, quando toda a } \\
\text { matéria prima utilizada em um experimento é } \\
\text { proveniente de um mesmo lote, ou há apenas um }\end{array}$ & $\begin{array}{c}\text { Montgomery (2009), Hamada (2009), } \\
\text { operador responsável pelo experimento, ou é utilizada } \\
\text { somente uma máquina durante os testes, entre outras }\end{array}$ & $\begin{array}{c}\text { Kempthorne (2008), } \\
\text { Kox, Hunter e Hunter }\end{array}$ \\
$\begin{array}{c}\text { Delineamento } \\
\text { em blocos } \\
\text { casualizados } \\
\text { (DBC) }\end{array}$ & $\begin{array}{c}\text { Os tratamentos são } \\
\text { aleatorizados }\end{array}$ & $\begin{array}{c}\text { Quando sabe-se, ou suspeita-se, que existam fontes de } \\
\text { variações indesejáveis, que podem ser causadas de lote } \\
\text { para lote, dia para dia, turno para turno, máquina para } \\
\text { (2005) e Mason, Gunst } \\
\text { máquina, operador para operador, etc }\end{array}$ & e Hess (2003) \\
\hline
\end{tabular}

Fonte: Autoria própria (2013)

Considerando limitações práticas na aplicação de tratamentos a unidades experimentais, pode ser necessária a atribuição de alguns fatores a unidades experimentais maiores - denominadas de parcelas principais - em comparação a outros fatores, que são destinados a parcelas 
experimentais menores - denominadas de subparcelas. Ressalta-se que nessas situações as parcelas principais são decompostas em subparcelas.

As possíveis restrições na atribuição dos tratamentos às parcelas experimentais estabelecem os esquemas fatoriais em parcelas subdivididas. Na Tabela 2 há um resumo com algumas dessas possibilidades de planejamento de experimentos.

Tabela 2 - Experimentos planejados resultantes da combinação de aspectos das unidades experimentais e número de fatores considerados

\begin{tabular}{|c|c|c|}
\hline \multirow{2}{*}{ Número de fatores } & \multicolumn{2}{|c|}{ Aspectos das unidades experimentais } \\
\hline & Homogêneas & Heterogêneas \\
\hline Um fator & Delineamento inteiramente casualizado & $\begin{array}{c}\text { Delineamento em blocos casualizados, } \\
\text { quadrado latino, quadrado greco-latino e } \\
\text { outros }\end{array}$ \\
\hline Dois ou mais fatores & $\begin{array}{l}\text { Delineamento inteiramente casualizado } \\
\text { em esquema fatorial }\end{array}$ & $\begin{array}{l}\text { Delineamento em blocos casualizados, } \\
\text { quadrado latino, quadrado greco-latino e } \\
\text { outros em esquema fatorial }\end{array}$ \\
\hline $\begin{array}{l}\text { Dois ou mais fatores em } \\
\text { esquema de parcela subdividida } \\
\text { (não abordados neste artigo) }\end{array}$ & $\begin{array}{l}\text { Delineamento inteiramente casualizado } \\
\text { em esquema de parcela subdividida }\end{array}$ & $\begin{array}{c}\text { Delineamento em blocos casualizados, } \\
\text { quadrado latino, quadrado greco-latino e } \\
\text { outros em esquema de parcela } \\
\text { subdividida }\end{array}$ \\
\hline
\end{tabular}

Fonte: Autoria própria (2013)

Quando os princípios citados não são incorporados ao projeto de um experimento e a aleatorização dos tratamentos nas unidades experimentais não é feita de forma adequada, os resultados das análises estatísticas podem ser inconclusivos, ou, o que é pior, enganosos (MASON; GUNST; HESS, 2003).

Galdamez e Carpinetti (2004) esclarecem que os experimentos conduzidos nas indústrias têm o intuito em melhorar o desempenho dos produtos e os processos de fabricação. Sendo que a melhoria dos processos pode ser obtida, conforme Montgomery (2004), por:

a) determinar quais são as variáveis mais influentes em um processo, por exemplo no planejamento de produtos novos;

b) determinar o valor a ser atribuído às variáveis influentes de modo que a variável resposta esteja próxima da exigência nominal;

c) determinar o valor a ser atribuído às variáveis influentes de modo que a variabilidade da variável resposta seja pequena;

d) determinar o valor a ser atribuído às variáveis influentes de modo que os efeitos das variáveis não controláveis sejam minimizados. 


\subsection{Experimento fatorial $2^{k}$ versus um-fator-por-vez}

Quando o interesse da indústria é estudar o efeito de mais de um fator, Tanco et al (2008) e Czitrom (1999) afirmam que a estratégia de experimentação muitas vezes considerada é a um-fatorpor-vez.

A estratégia de experimentação um-fator-por-vez consiste em modificar os níveis de um fator específico a cada teste, enquanto os demais permanecem fixos (MONTGOMERY, 2009; WU; HAMADA, 2009; CZITROM, 1999; DANIEL, 1994). Detalhes do desenvolvimento dessa estratégia para três e quatro fatores, todos de dois níveis, são apresentadas por Daniel (1994).

Uma crítica à estratégia um-fator-por-vez é que os efeitos fatoriais, principalmente das interações, não são estimados satisfatoriamente (SUDARSANAM; FREY, 2011; BOX; HUNTER; HUNTER, 2005; MONTGOMERY, 2004). Segundo Montgomery (2009) e Mason, Gunst e Hess (2003), o efeito de interação ocorre quando o efeito de um fator sobre a variável resposta depende dos níveis de outros fatores.

Alternativamente ao uso da estratégia um-fator-por-vez no estudo da influência de dois ou mais fatores na variável resposta, indica-se o planejamento de experimentos fatoriais (BOX; HUNTER; HUNTER, 2005, MONTGOMERY, 2004, CZITROM, 1999).

Segundo Wu e Hamada (2009) e Czitrom (1999), planejar um experimento fatorial, ao invés de um-fator-por-vez, é a maneira mais eficaz de determinar a influência de dois ou mais fatores sobre a variável resposta, por que:

a) requer menos recursos (experimentos, tempo, material) para a quantidade de informação obtida;

b) as estimativas dos efeitos fatoriais são mais precisas. Usando mais observações para estimar um efeito resulta em maior precisão;

c) o efeito das interações entre os fatores podem ser estimadas sistematicamente;

d) obtêm-se informações para uma região experimental maior.

De acordo com Wu e Hamada (2009), em experimentos fatoriais obtêm-se informações para uma região experimental maior porque cada efeito fatorial é calculado sobre todas as combinações possíveis dos níveis dos outros fatores, o que não necessariamente ocorre com a estratégia um-fatorpor-vez.

Alguns tipos especiais de planejamentos fatoriais são muito úteis no desenvolvimento e melhoria de processos. Um deles é o planejamento com $k$ fatores $(k \in \mathbb{N})$, cada um com dois níveis, que podem ser quantitativos - como valores de temperatura, pressão ou tempo - ou qualitativos - 
tipo de máquina ou operador (MONTGOMERY, 2009). Experimentos com $k$ fatores e todos com dois níveis são representados por $2^{k}$.

Geralmente, os níveis de cada fator em um experimento $2^{k}$ são chamados de "baixo" e "alto", ou "ausente" e "presente", e podem ser representados por “-" e "+", ou "0" e "1", ou “-1" e “+1”, respectivamente (MONTGOMERY, 2009). Outra forma de representar os tratamentos é usando letras minúsculas. Se uma letra está presente, então o fator correspondente é colocado no seu nível alto nesse tratamento; se a letra está ausente, o fator é colocado em seu nível baixo. O tratamento com todos os fatores no nível baixo é representado por (1) (MONTGOMERY, 2009).

Os planejamentos fatoriais $2^{k}$, conforme Box, Hunter e Hunter (2005), possuem algumas vantagens, entre elas a capacidade de proporcionar uma análise simplificada e, também, serem a base de muitos outros planejamentos úteis, como os fatoriais fracionados $2^{k-p}$. Os experimentos fatoriais fracionados são úteis quando o objetivo é examinar um grande número de fatores para determinar quais podem ser os mais importantes, ou quando tempo ou recurso disponível para o experimento são escassos, como ocorre no experimento de Aranda, Jung e Caten (2008), que utilizam o experimento fatorial fracionado $2^{5-1}$ na otimização de uma inovação tecnológica.

Nos experimentos fatoriais fracionados, alguns efeitos são confundidos entre si. O termo confundido é utilizado quando um ou mais efeitos não podem ser atribuídos de forma inequívoca para um único fator, interação ou bloco. Assim, é possível confundir efeitos entre fatores, como é o caso dos experimentos fatoriais fracionados e, também, confundir efeitos de fatores com os de blocos, que é o caso de confundimento em blocos. Os primeiros não serão abordados neste artigo. Detalhes sobre os fatoriais fracionados podem ser obtidos em Montgomery (2009), Box, Hunter e Hunter (2005), Hinkelmann e Kempthorne (2005), e no artigo clássico de Box e Hunter (1961).

\subsection{Experimento fatorial $2^{k}$ não repetido}

Conforme Montgomery (2009), os recursos disponíveis para experimentos são, geralmente, escassos, de tal forma que, quando o número de fatores é excessivo, é inviável realizar experimentos com repetições, a menos que o pesquisador esteja disposto a desconsiderar alguns fatores originais.

Experimentos fatoriais com uma só observação são denominados de fatoriais não repetidos. Uma abordagem para analisar esses experimentos, fatoriais não repetidos, consiste em considerar o princípio da escassez de efeitos, que é a suposição de que os efeitos das interações de ordem superiores são não significativos. Uma estimativa para o resíduo é o somatório das somas de quadrados dos efeitos considerados como insignificantes, e o restante da análise é conduzido normalmente. 
De acordo com Box, Hunter e Hunter (2005), ocasionalmente, efeitos de interações de alta ordem podem ser significativos, por isso mostra-se relevante um método preliminar para selecionálos. Após a identificação desses efeitos, eles são desconsiderados na estimação do resíduo.

Daniel (1959) propõe a utilização do gráfico de probabilidade semi-normal das estimativas dos efeitos, para verificá-los quanto a sua significância, em experimento fatorial $2^{k}$ não repetido. No entanto, Daniel (1976) e Box e Meyer (1986) admitiram que o gráfico de probabilidade normal é mais útil do que o semi-normal, pois, este último é menos capaz de revelar discrepância de pressuposições tais como a homocedasticidade.

A análise preliminar de um experimento fatorial não repetido não é realizada apenas pelo gráfico de probabilidade normal, há outros métodos menos triviais. Hamada e Balakrishnan (1998) analisam e discutem alguns desses métodos.

\subsection{A técnica do confundimento}

Há experimentos em que é impossível realizar uma repetição completa em blocos, pois a dimensão do bloco é menor do que o número total de tratamentos (MONTGOMERY, 2009). Uma alternativa para essa situação é a técnica do confundimento. Essa técnica faz com que a informação de alguns efeitos do experimento, em geral as interações de ordens superiores, sejam indistinguíveis dos efeitos de blocos.

O confundimento em blocos de um experimento fatorial $2^{k}$ pode ser realizado em $2^{p}$ blocos em cada repetição, com $p<k$. Montgomery (2009) e Winer (1962) descrevem um método de obter quais tratamentos serão designados para cada bloco. O método utiliza a combinação linear

$$
L=\alpha_{1} x_{1}+\cdots+\alpha_{k} x_{k}
$$

em que, $x_{i}$ é o nível do $i$ - ésimo fator que aparece em uma combinação de tratamentos particular e $\alpha_{i}$ é o nível do $i$ - ésimo fator para qual o efeito será confundido. Para experimentos $2^{k}$ tem-se $\alpha_{i}=1$ ou 0 e $x_{i}=1$ (nível alto) ou $x_{i}=0$ (nível baixo).

O método consiste em atribuir ao mesmo bloco os tratamentos que produzem o mesmo valor de $L$ congruente a 0 ou 1 módulo 2.

No planejamento de um experimento fatorial $2^{k}$ confundido em $2^{p}$ blocos, com $2^{k-p}$ tratamentos em cada bloco, selecionam-se $p$ efeitos independentes para que seus efeitos sejam confundidos com o efeito de blocos.

Além dos $p$ efeitos escolhidos inicialmente confundir-se-á $2^{p}-p-1$ efeitos de interações generalizadas. 
Os tratamentos que serão designados para cada um dos $p$ blocos são obtidos através de $p$ combinações lineares $L_{1}, \cdots, L_{p}$, tais como a da expressão (1). Com as $p$ combinações lineares formam-se $2^{p} p$ - uplas e todos os $2^{k-p}$ tratamentos de cada bloco produzem os mesmos valores $L_{1}(\bmod 2), \cdots, L_{p}(\bmod 2)$.

Conforme Hinkelmann e Kempthorne (2008), Garcia-Diaz e Phillips (1995) e Winer (1962), a soma de quadrados de blocos pode ser obtida através da expressão (2)

$$
S Q_{b l}=\frac{\sum_{j=1}^{J}\left(\sum_{i_{(j)}=1}^{I_{(j)}} Y_{i_{(j)} j}\right)^{2}}{I^{\prime}}-\frac{\left(\sum_{j=1}^{J} \sum_{i_{(j)}=1}^{I_{(j)}} Y_{i_{(j)} j}\right)^{2}}{I^{\prime} J}
$$

em que, $Y_{i_{(j)} j}$ é a observação do $i_{(j)}$ - ésimo tratamento dentro do $j$-ésimo bloco do experimento; $I^{\prime}$ é o total de tratamentos em cada bloco do experimento, ou seja, $I^{\prime}=2^{k-p} ; J$ é o total de blocos do experimento, ou seja, $J=n 2^{p}$, com $n$ o número de repetições.

A soma de quadrados de resíduos é obtida pela diferença da soma de quadrados total e as demais somas de quadrados, com exceção daquelas que são referentes aos efeitos confundidos com blocos.

Além deste artigo, Montgomery (2009), Hinkelmann e Kempthorne (2008), Garcia-Diaz e Phillips (1995) e Winer (1962) abordam a técnica do confundimento para experimentos fatoriais $2^{k}$.

\section{Materiais e métodos}

Os dados utilizados na ilustração das técnicas estatísticas de planejamento e análise de experimentos são provenientes de Santos (2007), e referem-se ao estudo da resistência mecânica de junções adesivas através de experimentos fatoriais $2^{k}$.

As unidades básicas do experimento são corpos de prova de junções adesivas que, conforme Santos (2007), foram obtidas por sobreposição simples de chapas de aço unidas por adesivo estrutural de base acrílica. A Figura 2 ilustra um corpo de prova, com as suas respectivas dimensões em $\mathrm{mm}$.

Figura 2 - Corpo de prova obtido através de sobreposição simples de chapas de aço

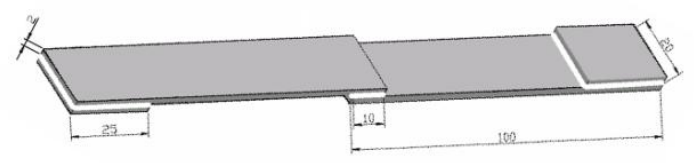

Fonte: Santos (2007) 
A variável resposta considerada é a tensão média de ruptura, obtida nos ensaios de cisalhamento por tração, que, segundo Santos (2007), são os mais utilizados na determinação da resistência de junções adesivas por sobreposição simples de chapas.

Santos (2007) considerou fatores que estão relacionados com a condição de cura do adesivo e de superfície do substrato, no caso as chapas de aço, todos em dois níveis. Os fatores e os seus níveis considerados foram: tratamento da superfície $(A)$, sem e com tratamento superficial utilizando o produto Bonderite NT1 da empresa Henkel; temperatura e tempo de cura $(B)$, nas condições $22^{\circ} C$ por 24 horas e $60^{\circ} C$ por 2 horas; rugosidade da superfície $(C)$, sem e com jateamento de areia; e uso de ativador $(D)$, sem e com ativador Loctite 7649 da empresa Henkel.

Para uma descrição mais detalhada do experimento aqui mencionado recomenda-se a apreciação de Santos (2007).

Os dados de Santos (2007) foram utilizados em três situações, sendo que em duas delas os dados foram adaptados.

A primeira situação refere-se à análise do experimento fatorial $2^{4}$ completo realizado em delineamento inteiramente casualizado com 10 repetições, tal como o de Santos (2007).

A justificativa para analisar o mesmo experimento nas mesmas condições apresentadas por Santos (2007) é fornecer o desenvolvimento da ANAVA, que não foi abordada diretamente pelo trabalho já citado.

Com o objetivo de exemplificar experimentos fatoriais não repetidos, na segunda situação é considerado, hipoteticamente, no experimento de resistência mecânica de junções adesivas, a impossibilidade de realizar mais do que uma repetição. Nessa situação as médias dos tratamentos da primeira situação foram consideradas como única observação de cada tratamento.

$\mathrm{Na}$ terceira situação, o experimento de resistência mecânica de junções adesivas será analisado como se tivesse sido planejado com dez repetições e quatro blocos para cada repetição. Sendo que a dimensão desses blocos é menor que a quantidade total de tratamentos do experimento.

Os blocos serão construídos a partir do confundimento dos efeitos independentes das interações $A C D$ e $B C D$ e o confundimento da interação generalizada $A B$.

A análise estatística para as situações 2 e 3 será apenas para verificar a significância dos efeitos principais, de interações e de blocos, sem considerar o desdobramento das interações caso estas sejam significativas. 


\section{Resultados e discussão}

\subsection{Situação 1: experimento fatorial $2^{4}$ completo}

O esquema da ANAVA para os testes referentes aos efeitos principais e de interações dos fatores tratamento da superfície $(A)$, temperatura e tempo de cura $(B)$, rugosidade da superfície $(C)$ e uso de ativador $(D)$, está apresentado na Tabela 3.

As primeiras conclusões que se obtém da ANAVA, Tabela 4, são que os efeitos principais $A, B, C$ e $D$ e os efeitos de interações $A C$ e $B C$ são estatisticamente significativos a um nível de $5 \%$ de significância.

Como nenhuma interação envolvendo o fator $D$ foi significativa, e sendo $C_{D}$ a estimativa do contraste para o efeito do fator $D$, verifica-se que o uso de ativador reduz a resistência mecânica das junções adesivas, pois, $C_{D}=-4,4413<0$. Sendo que o valor obtido desse contraste sugere que o uso do ativador Loctite 7649 da empresa Henkel deva ser evitado nas condições consideradas no experimento em destaque. Pois, em todas as combinações com os demais fatores, independente do nível, houve redução na resistência ao cisalhamento por tração nas junções adesivas.

$\mathrm{Na}$ Tabela 4 estão apresentadas as estimativas de todos os contrastes referentes aos efeitos principais e de interações dos fatores $A, B, C$ e $D$.

Tabela 3 - Esquema da análise de variância do experimento de resistência mecânica de junções adesivas em esquema

\begin{tabular}{lccccc}
\multicolumn{5}{c}{ fatorial $\mathbf{2}^{\mathbf{4}}$} \\
\hline Fonte de Variação & $\boldsymbol{G}$ & $\boldsymbol{S Q}$ & $\boldsymbol{Q M}$ & $\boldsymbol{F}$ & $\boldsymbol{v a l o r}-\boldsymbol{p}$ \\
\hline$A$ & 1 & 55,9323 & 55,9323 & 25,87 & $<0,01$ \\
$B$ & 1 & 196,2490 & 196,2490 & 90,75 & $<0,01$ \\
$C$ & 1 & 29,7390 & 29,7390 & 13,75 & $<0,01$ \\
$D$ & 1 & 788,9880 & 788,9880 & 364,86 & $<0,01$ \\
$A B$ & 1 & 0,0951 & 0,0951 & 0,04 & 0,83 \\
$A C$ & 1 & 10,5473 & 10,5473 & 4,88 & 0,03 \\
$A D$ & 1 & 1,2816 & 1,2816 & 0,59 & 0,44 \\
$B C$ & 1 & 13,5490 & 13,5490 & 6,27 & 0,01 \\
$B D$ & 1 & 0,9425 & 0,9425 & 0,44 & 0,51 \\
$C D$ & 1 & 6,3282 & 6,3282 & 2,93 & 0,09 \\
$A B C$ & 1 & 4,5765 & 4,5765 & 2,12 & 0,15 \\
$A B D$ & 1 & 2,1950 & 2,1950 & 1,02 & 0,32 \\
$A C D$ & 1 & 3,1136 & 3,1136 & 1,44 & 0,23 \\
$A B C D$ & 1 & 0,4796 & 0,4796 & 0,22 & 0,64 \\
Resíduo & 1 & 0,0093 & 0,0093 & 0,004 & 0,95 \\
\hline Total & 144 & 311,3944 & 2,1625 & & \\
\hline
\end{tabular}

Fonte: Autoria própria (2013)

Tabela 4 - Estimativas dos contrastes referentes aos efeitos principais e de interações dos fatores A, B, C e D

\begin{tabular}{lllll}
\hline$C_{A}=-1,1825$ & $C_{D}=-4,4413$ & $C_{A D}=0,1790$ & $C_{C D}=0,3978$ & $C_{A C D}=0,2790$ \\
$C_{B}=2,2150$ & $C_{A B}=-0,0488$ & $C_{B C}=-0,5820$ & $C_{A B C}=0,3383$ & $C_{B C D}=0,1095$ \\
$C_{C}=0,8623$ & $C_{A C}=-0,5135$ & $C_{B D}=-0,1535$ & $C_{A B D}=-0,2343$ & $C_{A B C D}=-0,0153$ \\
\hline
\end{tabular}

Fonte: Autoria própria (2013) 
A análise da influência dos fatores $A, B$ e $C$ dar-se-á pelo desdobramento das interações $A C$ e $B C$, em particular, dentro de cada nível do fator $C$.

As estimativas dos contrastes referentes aos desdobramentos das interações $A C$ e $B C$, em efeitos dos fatores $A$ e $B$ em cada nível do fator $C$, são: $C_{A: C_{-}}=-0,6690 ; C_{A: C_{+}}=-1,6960$; $C_{B: C_{-}}=2,7970 ; C_{B: C_{+}}=1,6330$.

Os esquemas das ANAVA para os testes referentes aos efeitos dos fatores $A$ e $B$ dentro de cada nível do fator $C$ estão apresentados, respectivamente, nas Tabelas 5 e 6 .

Nas ANAVA esquematizadas nas Tabelas 5 e 6 , todos os efeitos analisados são estatisticamente significativos a um nível de 5\% de significância, $F_{(1,144)}=3,91$.

Tabela 5 - Esquema da ANAVA do desdobramento da interação $\boldsymbol{A C}$ em efeito do fator $\boldsymbol{A}$ em cada nível do fator $\boldsymbol{C}$

\begin{tabular}{lccccc}
\hline Fonte de Variação & $G L$ & $S Q$ & $Q M$ & $F_{c}$ & valor $-p$ \\
\hline$A: C_{-}$ & 1 & 8,9512 & 8,9512 & 4,14 & 0,04 \\
$A: C_{+}$ & 1 & 57,5283 & 57,5283 & 26,60 & $<0,01$ \\
Resíduo & 144 & 311,3944 & 2,1625 & & \\
\hline Fon & & & &
\end{tabular}

Fonte: Autoria própria (2013)

Tabela 6 - Esquema da ANAVA do desdobramento da interação $\boldsymbol{B} \boldsymbol{C}$ em efeito do fator $\boldsymbol{B}$ em cada nível do fator $\boldsymbol{C}$

\begin{tabular}{lccccc}
\hline Fonte de Variação & $G L$ & $S Q$ & $Q M$ & $F_{c}$ & valor $-p$ \\
\hline$B: C_{-}$ & 1 & 156,4642 & 156,4642 & 72,35 & $<0,01$ \\
$B: C_{+}$ & 1 & 53,3338 & 53,3338 & 24,66 & $<0,01$ \\
Resíduo & 144 & 311,3944 & 2,1625 & & \\
\hline
\end{tabular}

Fonte: Autoria própria (2013)

Para o efeito da interação $A C$ na resistência mecânica de junções adesivas e o nível do fator $C$ fixado em baixo, a resistência mecânica das junções adesivas é reduzida quando considerado nível alto ao invés de baixo para o fator $A$, pois, $C_{A: C_{-}}=-0,6690<0$. Fixando o nível do fator $C$ em alto, o efeito do fator $A$ é semelhante ao caso anterior, no entanto, a redução na resistência mecânica das junções adesivas é mais expressiva ao considerar o nível alto ao invés de baixo, pois, $C_{A: C_{+}}=-1,6960<0$. O que significa que, desconsiderados os outros fatores, independente da rugosidade, a realização do tratamento superficial não é indicada, pois promove a redução da resistência do adesivo acrílico, sendo essa diminuição em maior escala quando houve jateamento na superfície em análise.

Por outro lado, Saleema et al (2012), em estudo de tratamento de superfícies simples de alumínio AA 6061 para aplicação de adesivos estruturais, verificam que mergulhar substratos de alumínio em uma solução muito diluída de hidróxido de sódio $(\mathrm{NaOH})$ pode ser considerado como um meio simples e eficaz de tratamento de superfície para junções adesivas usando os adesivos epoxídicos. 
Para o efeito da interação $B C$, quando o nível do fator $C$ está fixado em baixo, há um expressivo aumento na resistência mecânica das junções adesivas quando considerado o nível alto ao invés de baixo para o fator $B$, pois, $C_{B: C_{-}}=2,7970>0$. Fixando o nível do fator $C$ em alto, o efeito do fator $B$ é semelhante ao caso anterior, mas, o aumento na resistência mecânica é menos expressivo ao considerar o nível alto ao invés de baixo, pois, $C_{B: C_{+}}=1,6330>0$. Tais resultados sugerem que, desprezadas as influências dos outros fatores, independente da rugosidade, a cura que promove uma maior resistência ao cisalhamento por tração é aquela realizada a $60^{\circ} \mathrm{C}$ e $2 \mathrm{~h}$, corroborando com os resultados obtidos por Lin et al (2011), em que a temperatura de cura é um fator relevante na resistência mecânica de junções adesivas.

Em síntese, tem-se um aumento na resistência mecânica quando considerado o fator tratamento superficial $(A)$ no nível sem tratamento $\left(A_{-}\right)$combinado com qualquer nível do fator rugosidade $(C)$. No entanto, dentro do nível alto do fator rugosidade (com jateamento), o aumento da resistência mecânica é mais expressivo. Para o fator temperatura e tempo de cura $(B)$, aumentase a resistência mecânica quando considerado $60^{\circ} \mathrm{C}$ e 2 horas $\left(B_{+}\right)$dentro de cada nível do fator rugosidade, sendo esse aumento mais expressivo quando considerado o jateamento.

Considerando todas as combinações possíveis entre os níveis dos fatores, e o custo em obter junções adesivas com maiores resistências, é preferível considerar o fator tratamento superficial no nível baixo (sem tratamento), o fator temperatura e tempo de cura no nível alto ( $60^{\circ} C$ e 2 horas), o fator rugosidade no nível baixo (sem jateamento) e o fator ativador no nível baixo (sem ativador). Para essa combinação dos níveis dos fatores tem-se o tratamento $b$, cuja média é igual a $\bar{Y}_{b}=$ 18,207. A depender do custo desse processo de jateamento, a utilização de uma superfície sem jateamento, com os outros fatores nos níveis citados acima, pode representar a melhor relação custo-benefício. Esta combinação representa, em relação aos valores médios, apenas cerca de $1 \%$ a menos que a configuração com o jateamento.

Os resultados para as interações $A C$ e $B C$ também podem ser notados analisando-se os gráficos de efeitos de interações, Figura 3. 
Figura 3 - Gráficos de interações dos fatores $\boldsymbol{A}$ (tratamento superficial) e $\boldsymbol{B}$ (temperatura e tempo de cura) em cada nível do fator $\boldsymbol{C}$ (rugosidade), considerando a codificação “-” sem tratamento e “+” com tratamento, para o fator $\boldsymbol{A}$; “-” $22^{\circ} \mathrm{C}$ e 24 horas e "+" $60^{\circ} \mathrm{C}$ e 2 horas, para o fator B; "-" sem jateamento e "+" com jateamento, para o fator

C.
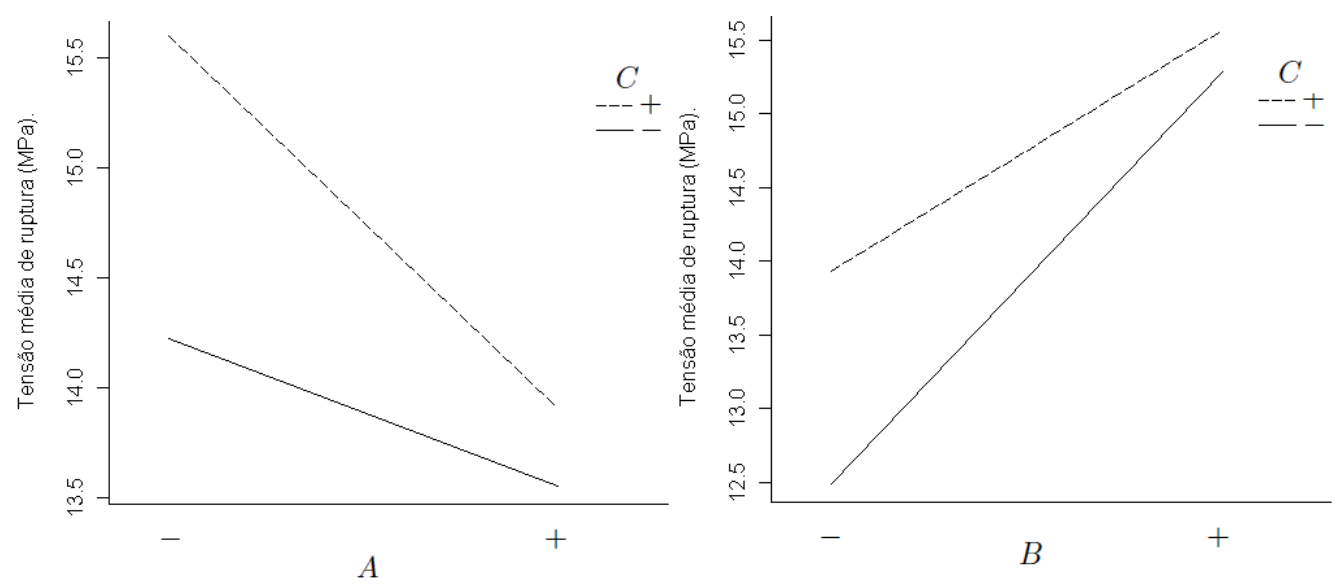

Fonte: Autoria própria (2013)

\subsection{Situação 2: experimento fatorial $2^{4}$ não repetido}

Já mencionado, na situação agora suposta, as médias dos tratamentos foram consideradas como única observação de cada tratamento.

O gráfico de probabilidade normal para os efeitos fatoriais do experimento agora considerado está ilustrado na Figura 4.

Figura 4 - Gráfico de probabilidade normal para os efeitos fatoriais do experimento fatorial não repetido de resistência mecânica de junções adesivas

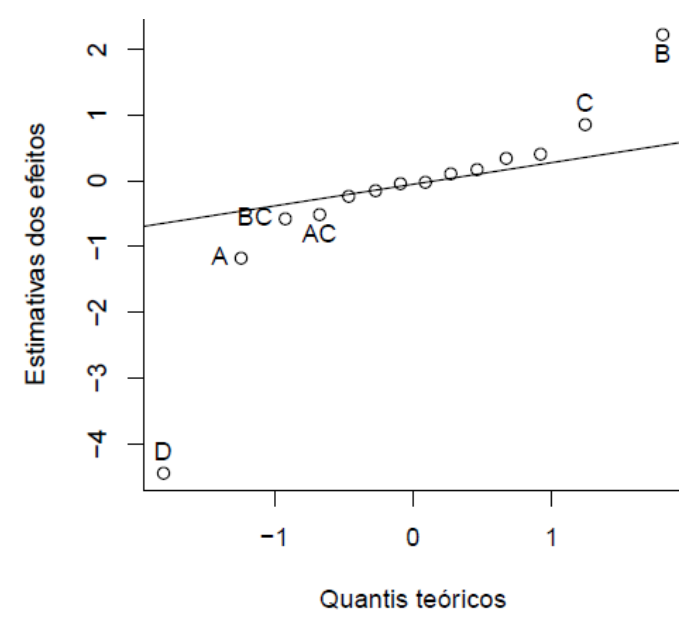

Fonte: Autoria própria (2013)

A análise do gráfico de probabilidade normal, Figura 4, leva a acreditar que as interações de ordem superior ou igual a 3 são estatisticamente não significativas. A combinação das somas de quadrados desses efeitos será considerada como uma estimativa da soma de quadrados de resíduos. 
Naturalmente, estarão associados 5 graus de liberdade ao resíduo, sendo 4 graus de liberdade das interações de ordem 3 e 1 grau de liberdade da interação de ordem 4.

Os valores de todas as estatísticas de testes para avaliar as hipóteses referentes à influência dos fatores $A, B, C$ e $D$ na resistência mecânica de junções adesivas estão apresentados na Tabela 7.

Tabela 7 - Esquema da ANAVA do experimento de resistência mecânica de junções adesivas em esquema fatorial $\mathbf{2}^{\mathbf{4}}$

\begin{tabular}{|c|c|c|c|c|c|}
\hline Fonte de Variação & $G L$ & $S Q$ & $Q M$ & $F$ & valor $-p$ \\
\hline$A$ & 1 & 5,5932 & 5,5932 & 25,96 & $<0,01$ \\
\hline$B$ & 1 & 19,6249 & 19,6249 & 94,58 & $<0,01$ \\
\hline C & 1 & 2,9739 & 2,9739 & 14,33 & 0,01 \\
\hline$D$ & 1 & 78,8988 & 78,8988 & 380,24 & $<0,01$ \\
\hline$A B$ & 1 & 0,0095 & 0,0095 & 0,05 & 0,83 \\
\hline$A C$ & 1 & 1,0547 & 1,0547 & 5,08 & 0,07 \\
\hline$A D$ & 1 & 0,1282 & 0,1282 & 0,62 & 0,47 \\
\hline$B C$ & 1 & 1,3549 & 1,3549 & 6,53 & 0,05 \\
\hline$B D$ & 1 & 0,0943 & 0,0943 & 0,45 & 0,53 \\
\hline$C D$ & 1 & 0,6328 & 0,6328 & 3,05 & 0,14 \\
\hline Resíduo & 5 & 1,0374 & 0,2075 & & \\
\hline Total & 15 & 111,4026 & & & \\
\hline
\end{tabular}

Fonte: Autoria própria (2013)

Na ANAVA esquematizada na Tabela 7, apenas os efeitos principais são significativos a um nível de $5 \%$ de significância, $F_{(1,5)}=6,61$. No entanto, nota-se que os níveis descritivos (valor $p$ ) dos fatores $A C$ e $B C$ são próximos de 0,05 , sugerindo resultados semelhantes do experimento completo, que já foram discutidos.

O planejamento de experimento fatorial não repetido é útil na escassez de unidades experimentais. No entanto, a não estimação direta do erro experimental, que é a finalidade da repetição, acarreta na obtenção de pouca informação comparado a experimentos repetidos.

\subsection{Situação 3: experimento fatorial $2^{4} \mathrm{com}$ alguns efeitos fatoriais confundidos com efeito de blocos}

Nesta situação, serão consideradas dez repetições e quatro blocos para cada repetição, com a dimensão dos blocos menor que a quantidade total de tratamentos do experimento.

Os efeitos das interações $A C D, B C D$ e $A B$ serão confundidos com os efeitos de blocos, sendo que as interações $A C D$ e $B C D$ são independentes e $A B$ é a interação generalizada obtida de $A C D$ e $B C D$.

Conforme a expressão (1), as combinações lineares para o confundimento dos efeitos das interações $A C D, B C D$ e $A B$ com o efeito de blocos são: $L_{A C D}=x_{1}+x_{3}+x_{4}$ e $L_{B C D}=x_{2}+x_{3}+$ $x_{4}$. Os valores de $L_{A C D}(\bmod 2)$ e $L_{B C D}(\bmod 2)$ e os tratamentos que de cada um dos 4 blocos, em cada repetição, estão apresentados na Tabela 8 . 
Tabela 8 - Tratamentos designados aos blocos de cada repetição do experimento de resistência mecânica de junções adesivas, para o confundimento dos efeitos $\boldsymbol{A} \boldsymbol{C D}, \boldsymbol{B} \boldsymbol{C} \boldsymbol{D}$ e $\boldsymbol{A B}$ com efeito de blocos

\begin{tabular}{cccc}
\hline Bloco 1 $(0,0)$ & Bloco 2 $(1,0)$ & Bloco 3 $(0,1)$ & Bloco 4 (1,1) \\
\hline (1) $c d a b c$ abd & $a b c$ bd acd & $b$ ac ad bcd & $c$ d ab abcd \\
\hline
\end{tabular}

Fonte: Autoria própria (2013)

Todas as estatísticas de teste, $F_{c}$, do experimento de resistência mecânica de junções adesivas, com os efeitos $A C D, B C D$ e $A B$ confundidos com efeito de blocos, estão apresentadas na Tabela 9, referente ao esquema da ANAVA.

Tabela 9 - Esquema da ANAVA do experimento de resistência mecânica de junções adesivas em esquema fatorial $\mathbf{2}^{\mathbf{4}}$ com os efeitos $\boldsymbol{A} \boldsymbol{C D}, \boldsymbol{B} \boldsymbol{C} \boldsymbol{D}$ e $\boldsymbol{A} \boldsymbol{B}$ confundidos com o efeito de blocos

\begin{tabular}{lccccc}
\hline Fonte de Variação & $G L$ & $S Q$ & $Q M$ & $F$ & valor $-p$ \\
\hline$A$ & 1 & 55,9323 & 55,9323 & 26,24 & $<0,01$ \\
$B$ & 1 & 196,2490 & 196,2490 & 92,07 & $<0,01$ \\
$C$ & 1 & 29,7390 & 29,7390 & 13,95 & $<0,01$ \\
$D$ & 1 & 788,9880 & 788,9880 & 370,16 & $<0,01$ \\
$A C$ & 1 & 10,5473 & 10,5473 & 4,95 & 0,03 \\
$A D$ & 1 & 1,2816 & 1,2816 & 0,60 & 0,44 \\
$B C$ & 1 & 13,5490 & 13,5490 & 6,36 & 0,01 \\
$B D$ & 1 & 0,9425 & 0,9425 & 0,44 & 0,51 \\
$C D$ & 1 & 6,3282 & 6,3282 & 2,97 & 0,09 \\
$A B C$ & 1 & 4,5765 & 4,5765 & 2,15 & 0,15 \\
$A B D$ & 1 & 2,1950 & 2,1950 & 1,03 & 0,32 \\
$A B C D$ & 1 & 0,0093 & 0,0093 & 0,004 & 0,95 \\
Blocos & 39 & 84,8801 & 2,1764 & 1,02 & 0,31 \\
Resíduo & 108 & 230,2027 & 2,1315 & & \\
\hline Total & 159 & 1425,4204 & & & \\
\hline Font Alat & & & & \\
\hline
\end{tabular}

Fonte: Autoria própria (2013)

Para um nível de 5\% de significância, o valor $F_{c}$ para os efeitos $A, B, C, D, A C$ e $B C$ é maior que $F_{(1,108)}=3,93$. Sendo assim, somente os testes referentes aos efeitos citados anteriormente são significativos, isto é, esses fatores são influentes na resistência mecânica de junções adesivas.

O teste $F$ para a influência do efeito de blocos na resistência mecânica das junções adesivas apresentou valor $F_{c}$ menor que o valor crítico de $F$ para o nível de $5 \%$ de significância, $F_{(39,108)}=$ 1,51, ou seja, não se pode afirmar que o fator controlado por blocos influencia na resistência mecânica das junções adesivas.

Note-se que os resultados agora obtidos são os mesmos da análise do experimento original. Com a ressalva que, quanto menor for a dimensão dos blocos, mais blocos por repetição serão necessários, acarretando em mais efeitos fatoriais confundidos com efeito de blocos e redução dos graus de liberdade do resíduo. 


\title{
5. Conclusão
}

Apesar de o estudo ter como referência ensaios destrutivos realizados em laboratório, é importante evidenciar a possibilidade de realização de experimentos dessa natureza ao longo da produção, ou seja, online. A identificação dos fatores influentes em um processo produtivo pode, e muitas vezes precisa ser, realizada sem a interrupção da produção.

$\mathrm{O}$ experimento fatorial $2^{k}$ mostrou-se um importante recurso para a melhor compreensão de um processo produtivo e a consequente implantação de melhorias contínuas. Além de sua proficuidade, este estudo evidencia a viabilidade desses planejamentos, inclusive em indústrias de pequeno e médio porte. Tal percepção tem fundamento na relativa facilidade de realização do experimento, tanto no relativo baixo custo, como no cálculo demandado para a obtenção dos importantes resultados.

Das três situações estudadas, observa-se que o confundimento de efeitos fatoriais com efeito de blocos mostrou-se uma solução intermediária para a experimentação na indústria quando o problema de esforço e custo é limitante.

\begin{abstract}
Experiments are conducted by industry in order to increase understanding of their manufacturing processes. Thus, this article aims to address the planning and analysis of $2^{k}$ factorial experiments in the industrial context. Specifically, we propose to expose aspects and basic concepts of experimentation and suggest as an alternative to the use of technical experimentation one-factor-ata-time, planning full factorial experiments at two levels, which are useful when there is a purpose of examining a large number of factors to determine which are the most important, or when time or stuff for the experiment are scarce. The techniques of confounding and unreplicated experiment are also discussed. The first is an alternative to experiments in which there is some difficulty in maintaining a constant feature of the production process, and the second when it is unfeasible performing repetitions. As a result are illustrated the statistical techniques presented. Applications were simulated from the same experiment to verify the influence of four factors on the mechanical strength of adhesive joints.
\end{abstract}

Key-words: confounding; industrial experiment; unreplicated experiment; one-factor-at-a-time.

\section{Referências}

ARANDA, M. H.; JUNG, C. F.; CATEN, C. S. t. Aplicação do projeto de experimentos para otimização de uma inovação tecnológica. Revista Gestão Industrial, v. 4, n. 2, p. 116-132, 2008.

BAKKE, H. A.; LEITE, A. S. M.; SILVA, L. B. Estatística multivariada: aplicação da análise fatorial na engenharia de produção. Revista Gestão Industrial, v. 4, n. 4, p. 01-14, 2008.

\section{cross ref}

BOX, G E. P. Evolutionary operation: a method for increasing industrial productivity. Journal of the Royal Statistical Society, v. 6, n. 2, pp. 81-101, 1957.

BOX, G. E. P.; HUNTER, J. S. The $2^{k-p}$ fractional factorial designs, part 1. Technometrics, v. 3, n. 3, p. 311-351, 1961. 
BOX, G. E. P.; HUNTER, J. S.; HUNTER, W. G. Statistics for experimenters: design, innovation, and discovery. 2. ed. Hoboken: John Wiley \& Sons, 2005. 633 p.

BOX, G. E. P.; MEYER, R. D. An analysis for unreplicated fractional factorials. Technometrics, v. 28, n. 1, p. 11-18, 1986.

\section{cross ref}

CASELLA, G.; BERGER, R. Inferência estatística. São Paulo: Cengage Learning, 2010. 588 p.

COCHRAN, W. G. Some consequences when the assumptions for the analysis of variance are not satisfied. Biometrics, v. 3, n. 1, p. 22-38, 1947.

\section{cross ${ }^{\text {ref }}$}

CZITROM, V. One-factor-at-a-time versus designed experiments. The American Statistician, v. 53, n. 2, p. 126-131, 1999.

DANIEL, C. Use of half-normal plots in interpreting factorial two-level experiments. Technometrics, v. 1, n. 4, p. 311341, 1959.

cross ref

DANIEL, C. Applications of statistics to industrial experimentation. New York: John Wiley \& Sons, 1976. 294 p. cross ${ }^{\text {ref }}$

DANIEL, C. Factorial one-factor-at-a-time experiments. The American Statistician, v. 48, n. 2, p. 132-135, 1994.

FISHER, R. A. The design of experiments. 9. ed. New York: HAFNER PRESS, 1971. 248 p.

GALDAMEZ, E. V. C.; CARPINETTI, L. C. R. Aplicação das técnicas de planejamento e análise de experimentos no processo de injeção plástica. Gest. Prod., v. 11, n. 1, p. 121-134, 2004.

cross ref

GARCIA-DIAZ, A.; PHILLIPS, D. T. Principles of experimental design and analysis. London: Chapman \& Hall, 1995. 409 p.

GOOS, P.; LANGHANS, I.; VANDEBROEK, M. Practical inference from industrial split-plot designs. Journal of Quality Technology, v. 38, n. 2, p. 162-179, 2006.

HAMADA, M.; BALAKRISHNAN, N. Analyzing unreplicated factorial experiments: a review with some new proposals. With discussions. Statistica Sinica, v. 8, n. 1, p. 1-41, 1998.

HINKELMANN, K.; KEMPTHORNE, O. Design and analysis of experiments: advanced experimental design. Hoboken: John Wiley \& Sons, 2005. 780 p.

cross ref

HINKELMANN, K.; KEMPTHORNE, O. Design and analysis of experiments: introduction to experimental design. Hoboken: John Wiley \& Sons, 2008. 631 p.

ILZARBE, L. et al. Practical applications of design of experiments in the fiel of engineering: a bibliographical review. Quality and Reliability Engineering International, v. 24, p. 417-428, 2008.

cross ref

IRWIN, J. O. Mathematical theorems involved in the analysis of variance. Journal of the Royal Statistical Society, v. 94, n. 2, p. 284-300, 1931.

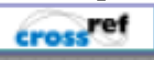


JONES, B.; NACHTSHEIM, C. J. Split-plot designs: what, why, and how. Journal of Quality Technology, v. 41, n. 4, p. 340-361, 2009.

LIMA, V. B. S.; BALESTRASSI, P. P.; PAIVA, A. P. Otimização do desempenho de amplificadores de radiofrequência banda larga: uma abordagem experimental. Produção, v. 21, n. 1, p. 118-131, 2011.

cross ref

LIN, J. et al A design of experiments assessment of moisture content in uncured adhesive on static strength of adhesivebonded galvanized SAE1006 steel. International Journal of Adhesion \& Adhesives, v. 31, p. 478-485, 2011.

cross ref

MASON, R. L.; GUNST, R. L.; HESS, J. L. Statistical design and analysis of experiments with applications to engineering and science. 2. ed. Hoboken: John Wiley \& Sons, 2003. 728 p.

cross ref

MONTGOMERY, D. C. Introdução ao controle estatístico da qualidade. Tradução Ana Maria Lima de Farias. 4. ed. Rio de Janeiro: LTC, 2004, 516p.

MONTGOMERY, D. C. Design and analysis of experiments. 7. ed. New York: John Wiley \& Sons, 2009.656 p.

SALEEMA, N. et al. A simple surface treatment and characterization of AA 6061 aluminum alloy surface for adhesive bonding applications. Applied Surface Science, v. 261, p. 742-748, 2012.

cross ref

SANTOS, D. J. Estudo experimental da resistência mecânica de junções adesivas. 2007. 140 p. Dissertação (Mestrado em Engenharia Mecânica) - Escola Politécnica da Universidade de São Paulo, São Paulo, 2007.

SUDARSANAM, N.; FREY, D. D. Using ensemble techniques to advance adaptive one-factor-at-a-time experimentation. Quality and Reliability Engineering International, v. 27, n. 7, p. 947-957, 2011.

cross ${ }^{\text {ref }}$

TANCO, M. et al. How is experimentation carried out by companies? A survey of three european regions. Quality and Reliability Engineering International, v. 24, p. 973-981, 2008.

cross ref

WINER, B. J. Statistical principles in experimental design. New York: McGraw-Hill, 1962. 672 p.

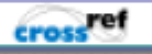

WU, C. F. J.; HAMADA, M. S. Experiments planning, analysis, and optimization. 2. ed., Hoboken: John Wiley \& Sons, 2009. $716 \mathrm{p}$.

\section{Dados dos autores:}

Nome completo: Juliano Bortolini

Filiação institucional: Universidade Federal de Mato Grosso - UFMT

Função ou cargo: Professor

Endereço completo para correspondência: Universidade Federal de Mato Grosso

Instituto de Ciências Exatas e da Terra, Departamento de Estatística, Avenida Fernando Corrêa da

Costa, no 2367, Boa Esperança, CEP: 78060-900 - Cuiabá, MT - Brasil.

Telefone para contato: 55-65-36158740 
Fax: 55-65-36158704

e-mail: julianobortolini@ufmt.br

Nome completo: Walter Accioly Costa Porto

Filiação institucional: Instituto Federal da Bahia - IFBA

Função ou cargo: Professor

Endereço completo para correspondência: Rua Guillard Muniz, 259 / 1101, Pituba. CEP: 41810-110

- Salvador, BA - Brasil.

Telefone para contato: 55-71-88348836

Fax: 55-71-33797796

e-mail:walterporto@hotmail.com

Nome completo: Marcelo Silva de Oliveira

Filiação institucional: Universidade Federal de Lavras - UFLA

Função ou cargo: Professor

Endereço completo para correspondência: Universidade Federal de Lavras, Departamento de

Ciências Exatas - Campus da UFLA, CEP 37200-000 - Lavras, MG - Brasil.

Telefone para contato: 55-35-38291373

e-mail:marcelo.oliveira@dex.ufla.br

Enviado em: 03/04/2013

Aprovado em: 10/06/2013 